\title{
The Grain Production Potential Assessment With Multiple Cropping Index (MCI) in China
}

\author{
Zhiqiang Gao $^{* \mathrm{a}, \mathrm{b}}$, Jicai Ning ${ }^{\mathrm{a}}$, Wei Gao \\ ${ }^{a}$ Yantai Institute of Coastal Zone Research, Chinese Academy of Sciences, Yantai, 264003, China \\ ${ }^{b}$ USDA UV-B Monitoring and Research Program and Center of Remote Sensing and Modeling for \\ Agricultural Sustainability, Natural Resource Ecology Laboratory, \\ Colorado State University, Fort Collins, CO, USA
}

\begin{abstract}
This paper retrieved the information of cropland and MCI (Multiple Cropping Index) of China in 2000 and 2009 with SPOT NDVI time series data and utilized meteorological data and statistical data released by the state to calculate potential MCI and statistical MCI. Then, the MCI potential of China and grain production potential based on MCI were calculated in order to analyze the potential spatial distribution characteristics of MCI and the potential spatial pattern characteristics. The national mean MCI potentials in 2000 and 2009 are 0.485 and 0.506 respectively calculated with the remote sensing method and statistical method. And the grain productivity potentials of China based on MCI are $51 \%$ and $53 \%$ respectively. The improvement of MCI potential not only increases hydrothermal utilization rate and the utilization rate of cropland but also enormously enhances the food security degree of China and provides more available cropland area for the economic development.
\end{abstract}

Keywords: China; Time Series Data; MCI; Change of Cropland; Grain Production Potential

\section{Introduction}

Multiple cropping is not only an important feature of cropping system of China but also an important approach to improve grain output and realize multiple operations. Multiple cropping is formed and developed according to natural (light, heat, water and soil), social and economic conditions. China spans eight temperature zones from north to south. As a result, different temperature zones have different heat conditions and growth periods, and the varieties and farming systems of crops in different zones also differ greatly. The cropping systems are also complicated and diversified (single cropping annually to triple cropping annually). Besides, the multiple methods like intercropping and interplanting exist. Multiple cropping can improve the utilization rate of natural resources like land, light and heat and human resources to increase grain output on the one hand; on the other hand, it can alleviate the contradiction of land fighting among grains, economic crops, green manure, etc. to some extend and promote the agricultural development (Fu, et al., 2001, Liu, et al., 1993, Yang, et al., 2000).

Multiple Cropping Index (MCI) has reflected the degree of utilizing agricultural resources for agricultural production in terms of time scale. The essence of MCI is to reflect the utilization condition and degree of the cropland by adopting a certain cropping system along the time series from the perspective of the number of times of utilizing cropland. It is a fundamental index used to measure the intensive utilization degree of cropland resources in the study of cropping systems. It is also a quantified expression of multiple-cropping degree (Liu, et al., 1990, 1996).

*Corresponding author. Email: zqgao@yic.ac.cn

Remote Sensing and Modeling of Ecosystems for Sustainability XI, edited by Wei Gao, Ni-Bin Chang, Jinnian Wang, Proc. of SPIE Vol. 9221,922116

(c) 2014 SPIE · CCC code: 0277-786X/14/\$18 - doi: 10.1117/12.2058304 
According to the current situation of domestic researches on the dynamic changes of cropland and MCI with remote sensing, Then the potential of MCI in China combining the meteorological data is calculated with the potential model of MCI.

\section{Methodology}

\subsection{Data Processing}

In this paper, the SPOT VEGETATION NDVI data is used and the NDVI data during 2000 to 2009 is selected in two periods, the 36 VGT-S10 files are included in each year. Then the images are stacked to form annual NDVI time series data sets of 36 wave bands according to annual stacking. The change threshold algorithm (according to the suggestion of Zhang Jixian, it will be rated as an abnormal value if the change range exceeds $20 \%$ of the reference value) and sliding window algorithm with the step of 3 are adopted in this study for filtering. The filtering makes the NDVI time series data closer to the true condition and the curve "smoother". The information of land use/land cover and MCI in China may be retrieved with the time series data.

Meanwhile, the annual mean values of precipitation and temperature from 670 meteorological stations under China Meteorological Administration over the years are used in the paper. The spatial data is generated by means of interpolation to calculate the theoretic MCI (multiple cropping index potential) of China. And, the crop outputs, crop seeded area and cropland area of provinces of China in 2000 and 2009 are gathered by National Bureau of Statistics of China (National Bureau of Statistics, 2001, 2010) to calculate the MCI and the grain production potential based on MCI of each province in China.

\subsection{Calculation of Grain Production Potential Based on MCI}

The MCI is of great importance for the agricultural production. It reflects the conditions of comprehensive utilization of cropland and resources like light, heat and water for cultivation of crops. During the growth and development of any crops, certain water and heat conditions are required. 100-300 days of growth period, $1000-3000$ of accumulated temperature and $300-800 \mathrm{~mm}$ of precipitation are the basic requirements for their growth and maturity. Otherwise, the crops cannot complete the whole life. Only when the growth period, accumulated temperature and precipitation of a region exceed the needs of its leading crops, other crops can be planted according to residual growth period, accumulated temperature and precipitation. Therefore, the hydrothermal condition is a basic factor influencing the value of MCI (Fan, et al., 2003, 2004, Gu, et al., 2003).

The relational model between MCI and water\& heat is shown as follows according to the basic conditions required for the crop growth above:

$$
\begin{array}{r}
M_{T}=\left\{\begin{array}{lr}
100 & T<3400 \\
(T-3400) * 0.125+100 & 3400 \leq T \leq 4200 \\
200 & 4200 \leq T \leq 5200 \\
(T-5200) * 0.1+200 & 4200 \leq T \leq 5200 \\
300 & T>6200
\end{array}\right. \\
M_{R}=\left\{\begin{array}{lr}
100 & R<500 \\
(R-500) * 0.14+200 & 500 \leq R \leq 1200 \\
300 & R>1200
\end{array}\right.
\end{array}
$$

MT refers to $\mathrm{MCI}$ potential based on accumulated temperature; $\mathrm{T}$ refers to the accumulated temperature above 0 ; MR refers to MCI potential based on precipitation; $\mathrm{R}$ refers to the annual average precipitation. Since accumulated temperature and precipitation are two factors restraining the improvement of MCI and the MCI potential becomes low when either factor constrains much, the MCI potential is the minimal value simulated by two models. Therefore, the MCI potential model obtained is as follows (the minimal value of the two is obtained). 


$$
M C I_{\text {potential }}=\min \left(M_{T} \text {.or. } M_{R}\right)
$$

\section{Results and Discussion}

In regions of China where light, heat and water are sufficient, the cropping system has gradually developed to multiple cropping with the development of agricultural cropping technologies. Resources including light, heat and water can be fully utilized through multiple cropping, and the realistic MCI is the result of full utilization of light, heat and water in this area (Liu, 1993, 1996). The potential MCI (i.e. theoretic MCI) calculated by Formula 1 and Formula 2 above refers to the maximum MCI of each region calculated according to both the demand limit of crop growth for water and heat and the hydrothermal conditions of each region. Figure 1 indicates the regions of China with potential MCI of triple cropping are mainly distributed in subtropical zones in south of Yangtze River. The regions with potential MCI of double cropping are mainly distributed in the temperate zone of North China Plain, Sichuan Basin, etc. The regions with potential MCI of single cropping are mainly distributed in the north temperate zone of North China, which mainly includes Liaoning Province, Jilin Province, Heilongjiang Province, Inner Mongolia, Xinjiang, Shanxi Province, Shaanxi Province, etc.

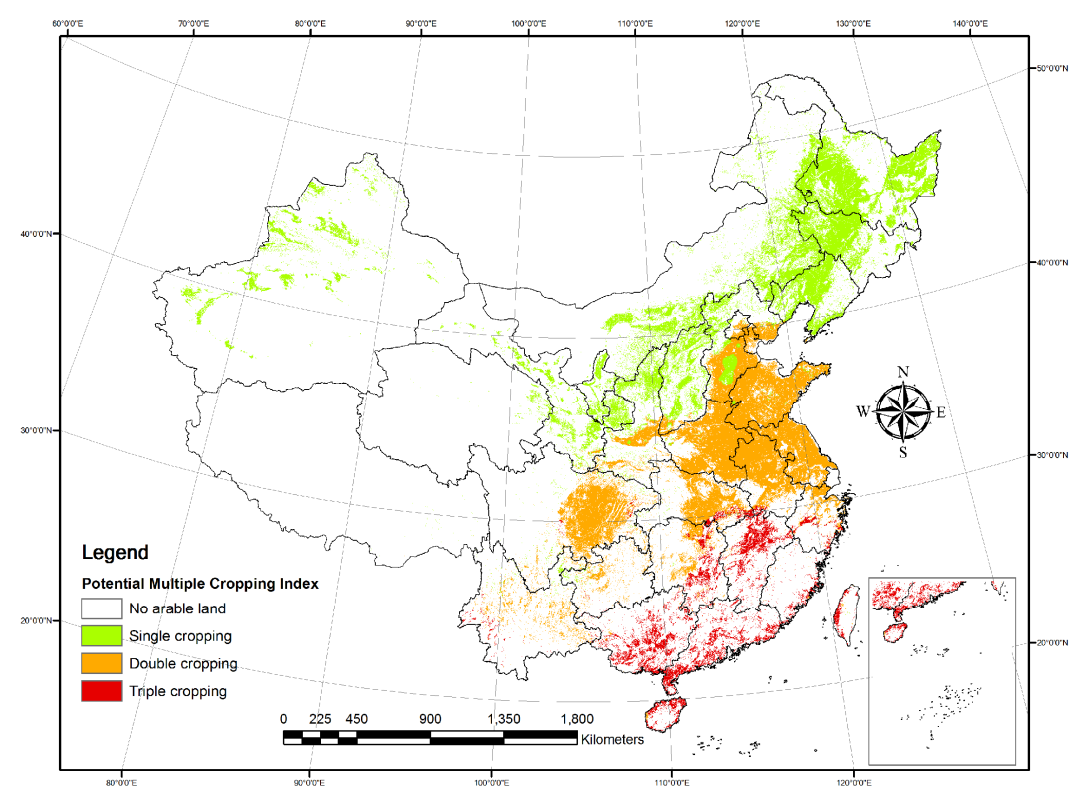

Figure 1. The Potential MCI by climate data

The MCI and potential MCI of each province in 2000 and 2009 are calculated by combining the MCI map (potential MCI data map) with the administrative boundary data of provinces in China. At the same time, the MCI of each province in 2000 and 2009 are calculated with the cropland area and crop seeded area of each province in 2000 and 2009 collected by National Bureau of Statistics in 2001 and 2010. Although the MCI of each province obtained from the three methods (Remote sensing, Meteorological and Statistics) differs, the cropping rules of China under the hydrothermal control has been reflected.

The potential MCI of China is taken as the benchmark. Then, the MCI potential of each province can be obtained by the potential MCI deducting the MCI in 2009 by the method of remote sensing (Figure 2).

Figure 2 indicates the distribution of MCI potential of China as follows: the provinces with multiple cropping potential above single cropping mainly include Fujian Province, Jiangxi Province, Hunan 
Province, Hubei Province and Sichuan province which locate in southern temperate zone and northern subtropical zone. The provinces with multiple cropping potential around single cropping mainly include Guangdong Province, Guangxi Province, Hainan Province, Taiwan, Zhejiang Province, Shanghai, Yunnan Province, Guizhou Province, etc. The provinces with MCI potential around semi-single cropping mainly include Jiangsu Province, Anhui Province, Henan Province, Hebei Province, Shandong Province, Beijing, Tianjin and Liaoning Province. The multiple cropping potential is close to 0 in other northern provinces like Heilongjiang Province, Jilin Province, Inner Mongolia, Xinjiang, Qinghai Province, Tibet, Ningxia, Gansu Province, Shanxi Province and Shaanxi Province due to the limitation of water and heat. The national MCI potential is calculated as 0.453 , which indicates that abundant water and heat in provinces of South China make great space for further improvement of cropping system. In other words, the multiple cropping potential is relatively big.

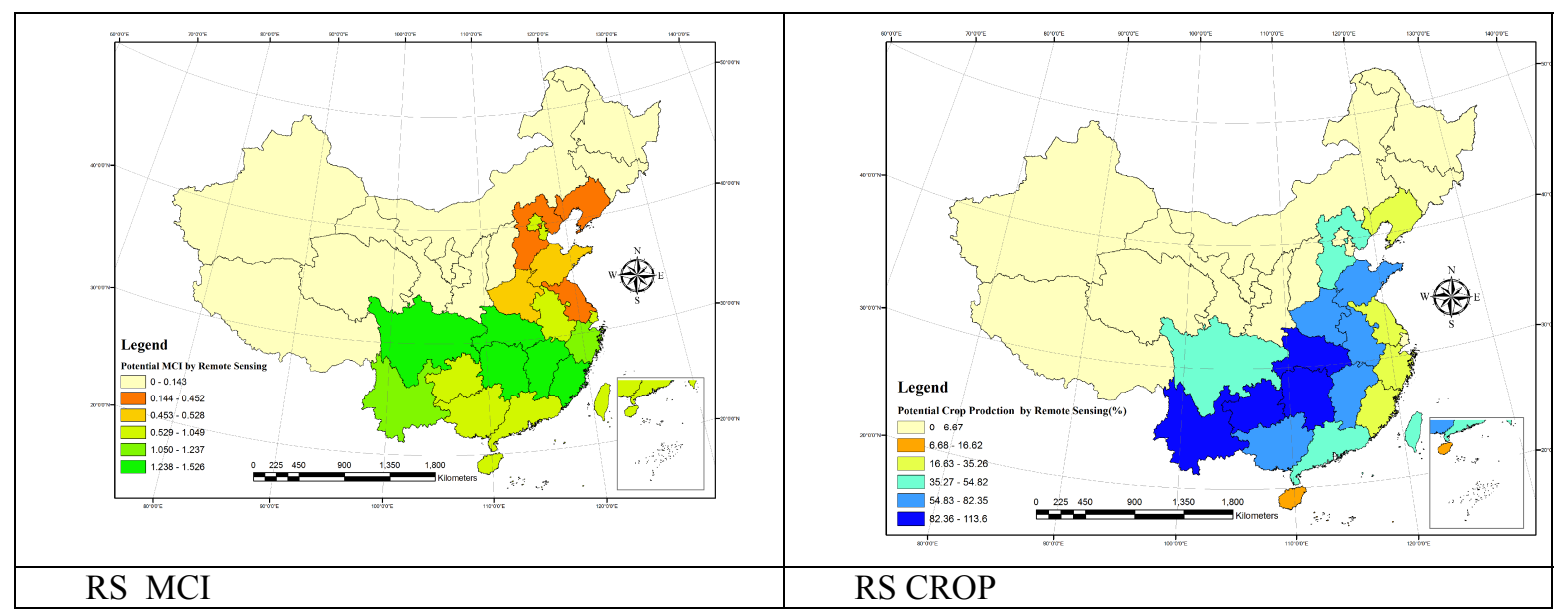

Figure 2. Distribution Maps of Potential MCI and Grain Production Potential of China by SPOT NDVI

\section{Conclusions}

This paper retrived the cropland information of China in 2000 and 2009 by means of SAM supervised classification with SPOT NDVI time series data. Meanwhile, the noises of SPOT NDVI time series data were eliminated by combining curve-fitting with HANTS (Harmonic Analysis of Time Series) of Fourier transformation technology. The MCI of China in 2000 and 2009 was extracted by reconstruct the crop growth curve.

The calculated average MCI potential of China is 0.506 (statistical) and 0.485 (remote sensing) while the grain production potential based on MCI of China is 51\% (statistical) and 53\% (remote sensing). Regions with big grain production potential based on MCI are located in the Southeast China, east of $400 \mathrm{~mm}$ precipitation line of China. Due to the limitation of precipitation and temperature, West China and North China have limited grain production potential based on MCI.

The grain production potential of China based on MCI is above $50 \%$. If $10 \%$ of the grain production potential is turned to actual output, it will be equal to the increase of the actual grain output of about 100 million mu cropland in China. The improvement of MCI potential not only increases hydrothermal utilization rate and the utilization rate of cropland but also enhances the food security degree of China enormously. 


\section{Acknowledgements}

The author are grateful for the support from Key Research Program of the Chinese Academy of Sciences( KZZD-EW-14),National Natural Science Foundation of China (41171334), Talent Fund of Yantai Institute of Coastal Zone Research, Ecological Innovation \& Breeding Project (Y254021031,Y355031061), and USDA NIFA Project (2010-34263-21075).

\section{References}

[1] Davis, A. R., Bush, C., Harvey, J. C. and Foley, M. F., "Fresnel lenses in rear projection displays," SID Int. Symp. Digest Tech. Papers 32(1), 934-937 (2001).

Book

[3] Booth, N. and Smith, A. S., [Infrared Detectors], Goodwin House Publishers, New York \& Boston, 241-248 (1997).

Fan, J.L., Wu,B.F., "A methodology for retrieving cropping index from NDVI profile," Journal of Remote Sencing, 8(6), 628-636(2004)

Fan ,J.L. "Study on monitoring methods for multiple cropping index. Journal of Remote Sensing," Dissertation of Institute of Remote Sensing Applications, 2003.

Fu ,Z.Q., Cai,Y.L., , Yang,Y.X.,. "Research on the relationship of cultivated land change and food security in China," Journal of Natural Resources, 2001, 16(4), 313-319(2001).

Gu Z.H., [A Study of Calculating Multiple Cropping Index of Crop in China Using SPOT/VGT MultiTemporal NDVI Data], Beijing Normal University, Beijing, 120-134(2003).

Liu Xunhao. "The view of Chinese cropping system in 1990s," Cultivate and Plant, 1992, (2):1-9. (in Chinese with English abstract)

Liu Xunhao. " Cropping System in China Beijing,": Agricultural Press, 1993. 12-15. (in Chinese with English abstract)

Liu Xunhao. "Cultivation, Beijing: China Agricultural Press, 1996. 1-9 (in Chinese with English abstract)

Yang H, Li X. Cultivated land and food supply in China. Land Use Policy, 2000, 17(2): 73-88 